\title{
Novel Mechanisms and Targets in Renal Cell Carcinoma Progression
}

\author{
Mark E Dockrell ${ }^{1,2 *}$, Robert G Price ${ }^{1,3}$ and Christer Hogstrand ${ }^{3}$ \\ ${ }^{1}$ SWT Institute for Renal Research, London, UK \\ ${ }^{2}$ St George's University of London, UK \\ ${ }^{3}$ King's College London, UK
}

*Corresponding author: Mark Dockrell, SWT Institute for Renal Research, Renal Unit, St Helier Hospital, Wrythe Lane, London, SM5 1AA, UK.

To Cite This Article: Mark E Dockrell, Novel Mechanisms and Targets in Renal Cell Carcinoma Progression. 2020 - 10(1). AJBSR.MS.ID.001463. DOI: 10.34297/AJBSR.2020.10.001463.

Received: 阱August 07, 2020; Published: 眥 August 20, 2020

\section{The Issue}

The consensus of The European Renal Cancer Roundtable meeting held in Brussels in 2019 was that the most pressing challenge is improved early detection. Each year sees around 400 000 new cases of kidney cancer worldwide. The 10 countries with the highest rates of kidney cancer are all in Europe; from Belarus to Iceland. Renal Cell Carcinoma (RCC) accounts for $80 \%$ of all kidney cancers. RCC are reported as a diverse group such as clear cell ( $-70 \%$ of the total) and of proximal tubule origin as well as papillary $(\sim 10 \%)$ and chromophobe $(\sim 5 \%)$, the latter 2 of distal tubule origin with a large number of gene mutations [1]. Yet, there are few causal genes driving RCC. The VHL gene is a common initiating mutation in Clear Cell RCC (ccRCC) but loss of VHL function is insufficient to develop ccRCC. There are environmental/behavioural associations with RCC including some of the usual suspects, smoking and obesity as well regular or prolonged acetaminophen use. When pre-metastatic RCC is treated, usually with surgery, 5- and 10-year survival rates are good. 5-year survival rates for metastatic RCC are generally poor, less than $10 \%$, despite the use of antiangiogenic tyrosine kinase inhibitors such as sunitinib. Unfortunately, due to substantial renal reserve more than $50 \%$ of RCC are detected incidentally and often when it has already spread [2].

\section{The Status Quo}

Tissue marker studies have produced some candidates but reliability and reproducibility of marker detection has proven problematic. Some urinary markers of general tubular damage have been proposed, such as Kim-1 and NGAL, but these are not specific for cancer. In our opinion, there is significant potential in looking at markers of cancer from other tissues which may have particular relevance in the proximal tubule.

\section{A Disruptive Perspective}

The ZIP family of zinc importers has 14 members, several of which have been associated with a variety of cancers. Among these ZIP10 is the best candidate; expression of ZIP10 mRNA has been found to be elevated in RCC compared with adjacent tissue, and to be significantly higher in high-grade clear cell RCC (ccRCC) compared to pre-metastatic ccRCC [3]. Data in the TGCA and Oncomine databases confirm that ZIP10 mRNA is upregulated in ccRCC, as well as in papillary RCC.

ZIP10 mRNA expression, which is induced by STAT3 and -5, is also overexpressed in metastatic breast cancer and lymphomas [4]. It dramatically increases proliferation, migration and invasive behaviour of breast cancer cells, where it forms a dimer with ZIP6. The ZIP10:ZIP6 dimer is required and sufficient to initiate mesenchymal-to-epithelial transition in cultured MCF7 cells. ZIP10 acts by initiating Zn2+-mediated activation of AKT and inhibition of GSK-3ß, triggering the adverse outcome pathway leading to cell growth and invasiveness [5]. Interestingly, blocking the ZIP10:ZIP6 heterodimer-mediated zinc influx by specific antibodies stops mitosis, confirming the requirement for this zinc influx as a mitosis trigger [6]. Zn2+ influx through ZIP transporters also leads to activation of Metal-Responsive Transcription Factor-1 (MTF1), which induces expression of several matrix metalloproteinases 
of significance for local invasiveness and metastasis of tumours including ccRCC [5].This mechanistic link between expression of ZIP proteins and cell aggressive behaviour make them interesting targets for pharmaceutical intervention. Anti-ZIP strategies are already in development (Figure 1).

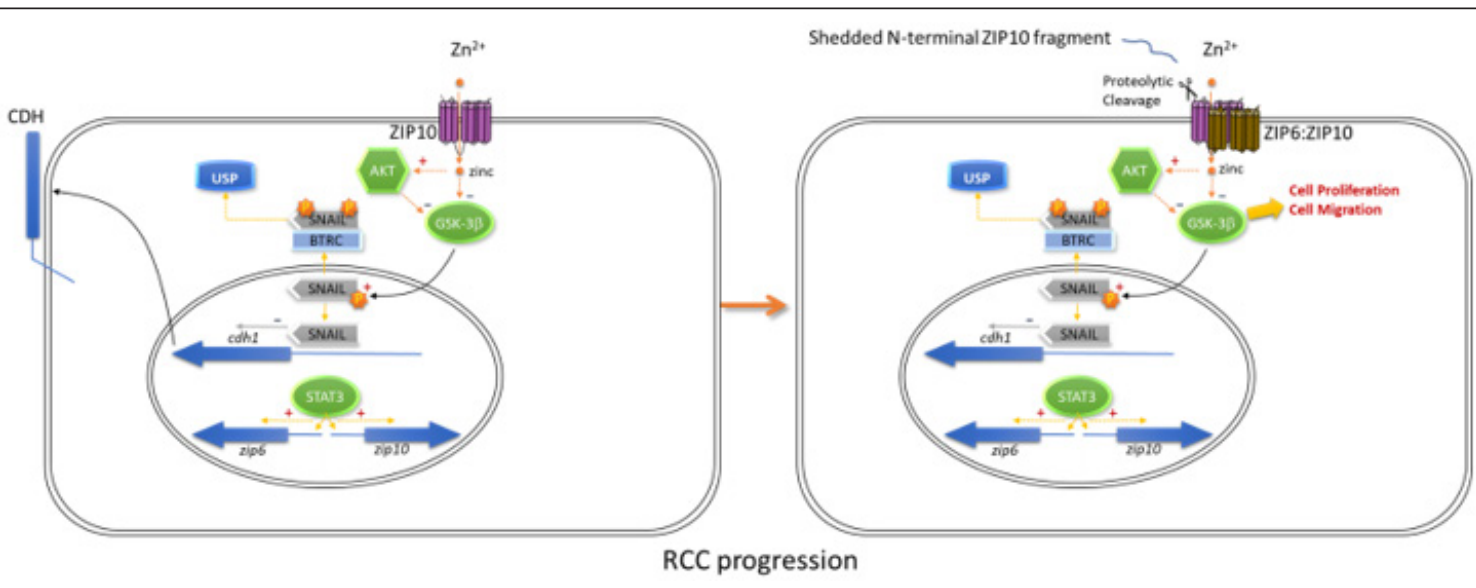

Figure 1: ZIP10 drives proliferation, migration, and metastasis of cells through activation of AKT and inhibition of GSK-3ß. The non-cancerous cell (1) can be transformed (2) by the upregulation of ZIP10 and its activation through ectodomain shedding, leading to blocked cadherin expression and driving a metastatic phenotype [6].

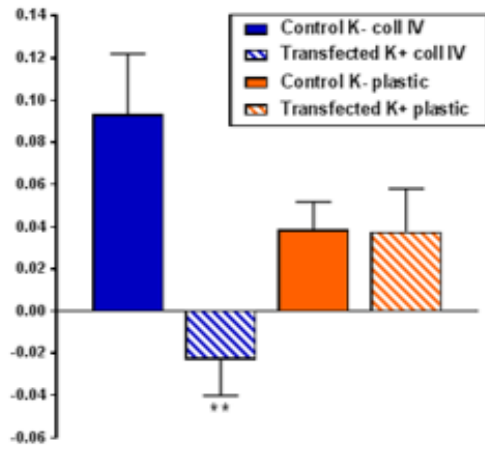

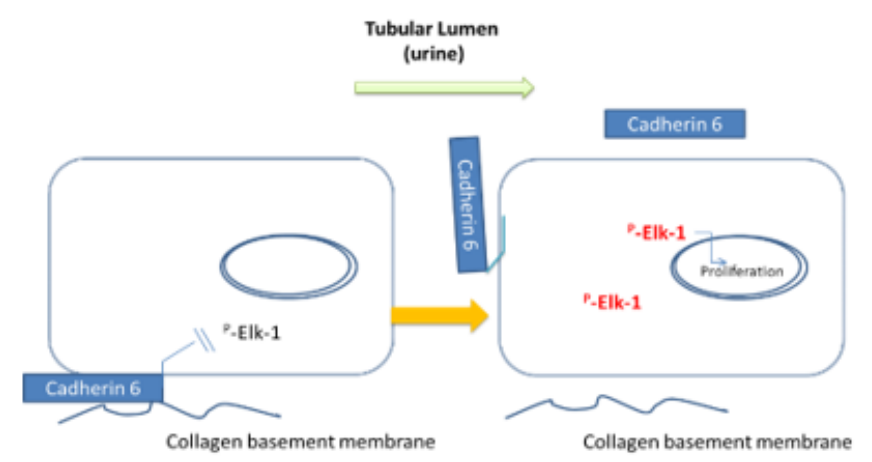

Figure 2: Cadherin 6 expression supresses Elk1 activation of cells grown on collagen IV . (A) Transformed human proximal epithelial cells (HKC8) were transfected with a vector containing cadherin 6 (hashed boxes) or an empty vector (solid boxes) and cultured on collagen IV (blue) or plastic (red) and incubated with TGF $\beta 1$ for 15 minutes. Cellular expression of phosphor Elk-1 was determined by western blotting. (B) Figurative representation of Cadherin 6 dissociation and Elk-1 activation.

There are clear advantages to investigating biomarkers that are part of the disease process rather than general indicators of dysfunction. The cadherins are a large family of transmembrane glycoproteins involved in embryogenesis and maintenance of epithelial integrity throughout life. Cadherin-mediated adhesion regulates cell growth and differentiation. Disruption of cadherin expression and signalling play key roles in tumorigenesis and tumor progression. E-cadherin (cadherin 1, CDH1) is the archetypal cadherin expressed widely throughout the body. Loss of E-cadherin expression predicts the loss of epithelial cell adhesion and is a prognostic factor for many cancers. Cadherin 6 (CDH6) is a class II cadherin and its upregulation reportedly promotes the motility and invasion of several cancer cells. However, in the healthy human proximal tubule epithelia there is no E-cadherin; there is cadherin
6 (K-cadherin). Disruption of Cadherin 6 in RCC was reported over 20 years ago [7]. Recent work from our team supports a role for Cadherin 6 in maintaining epithelial phenotype in primary human proximal tubule epithelial cells. Disruption of cadherin 6 expression was associated with an increase in potentially pro-cancerous intracellular signalling cascades including Elk1 and Erk5 (Figure 2).

Typically, cadherins are transmembrane proteins with three components: the large extracellular domains form homotypic cadherin-cadherin interactions, tethering neighbouring cells; a single-pass transmembrane domain; and a cytoplasmic domain with at least one phosphorylation site that regulates binding to catenins and subsequent binding to the actin cytoskeleton. The degree of force on the extracellular domain influences the binding 
to the cytoskeleton and hence the epithelium is held together through pressure dependent inside-out signalling. Unlike classical Type I and Type II cadherins cadherin 6 is expressed basally interacting with collagen in the basement membrane. When the interaction with collagen is altered either by the loss of this peculiar localisation or cleavage of the extracellular domain, the pro-oncogenic signalling is primed.

\section{Conclusion}

Biomarkers can be invaluable aids in disease management. Biomarkers can be used in diagnosis and in measuring the progress of disease; they can also be useful in evaluating the most effective therapeutic regimes. Ideally they should be accessible and measureable. Many biomarkers have been shown to have a close association with disease progression, at least in some populations. However, markers that have a role as mediators of the pathology are far more likely to be of real value. They also have the potential of identifying new targets for treatment. Both Cadherin 6 and ZIP 10 are integral parts of the oncogenic process in human proximal tubule epithelial cells and hence the nature of their expression is tied to the cellular fate. Furthermore, both ZIP10 and Cadherin 6 are potentially non-invasive urinary biomarkers, as they both have ectodomains that's are enzymatically cleaved and shed into the urine.

\section{References}

1. Valdair F Muglia, Adilson Prando (2015) Renal cell carcinoma: histological classification and correlation with imaging findings. Radiol Bras 48(3): 166-174

2. Zhongjun Li, Ping Hao, Qingjian Wu, Fengjie Li, Jiang Zhao, et al. (2016) Genetic mutations associated with metastatic clear cell renal cell carcinoma Oncotarget 7(13): 16172-16179.

3. Deeksha Pal, Ujjawal Sharma, Shrawan Kumar Singh, Rajendra Prasad (2014) Association between ZIP10 gene expression and tumor aggressiveness in renal cell carcinoma Gene 552(1): 195-198.

4. Kathryn M Taylor, Issa A Muraina, Dylan Brethour, Gerold Schmitt-Ulms, Thirayost Nimmanon, et al. (2015) Zinc transporter ZIP10 forms a heteromer with ZIP6 which regulates embryonic development and cell migration Biochem J 473(16): 2531-2544.

5. Jin-Hong Kim, Jimin Jeon, Minhee Shin, Yoonkyung Won, Minju Lee, et al. (2014) Regulation of the Catabolic Cascade in Osteoarthritis by the ZincZIP8-MTF1. Axis Cell 156(4):730-743.

6. Thirayost Nimmanon, Silvia Ziliotto, Olivia Ogle, Anna Burt, Julia MW Gee, et al. (2020) Novel zinc-mediated trigger for mitosis: the role of the ZIP6/ZIP10 heteromer in driving mitosis. Cell and Molecular Life Sciences, in press

7. Shimazui T, Oosterwijk E, Akaza H, Bringuier P, Ruijter E, van Berkel H, et al. (1998) Expression of cadherin-6 as a novel diagnostic tool to predict prognosis of patients with E-cadherin-absent renal cell carcinoma. Clin Cancer Res 4(10): 2419-2424. 